\section{Successful use of phlebotomy to treat severe secondary polycythemia due to chronic lung disease}

\author{
Jacob Fuqua, Josephine Reece, \\ Sarah Sofka \\ Department of Medicine, West Virginia \\ University, Morgantown, WV, USA
}

\begin{abstract}
Secondary polycythemia (SP) occurs as a result of increase erythropoietin levels most commonly as a result of tissue hypoxia. Symptoms such as erythromelalgia, pruritis, and bleeding, which are frequently seen in polycythemia vera (PV), do not commonly occur in SP. Phlebotomy is considered one of the mainstays of therapy for $\mathrm{PV}$ but is rarely used for treatment of SP due to concern about worsening tissue hypoxia. We present the case of a patient with severe SP due to chronic hypoxic lung disease who presented with erythromelalgia, pruritis, and bleeding and was treated successfully with therapeutic phlebotomy. This case illustrates the importance of considering the use of therapeutic phlebotomy in symptomatic patients with severe SP.
\end{abstract}

\section{Introduction}

Phlebotomy, also known as blood-letting or venesection, has been used for over five millennia to treat a multitude of illnesses. From febrile illnesses to heart failure, phlebotomy was used to treat inflammation that resulted from having excess blood in the body. ${ }^{1}$ Although most bygone indications for phlebotomy have safer and effective treatments, the process is still a mainstay of therapy for polycythemia vera (PV), porphyria cutanea tarda, and hemochromatosis. PV is a myeloproliferative disorder which results in erythrocytosis and thrombocytosis. Erythromelalgia, pruritis, and bleeding usually only occur in PV. Complications can include ischemic cardiovascular disease, stroke, and arterial or venous thromboembolism. ${ }^{2}$ Secondary polycythemia (SP) is a similar entity but occurs as a result of increased erythropoietin (EPO) levels. A common cause of SP is chronic hypoxia. In most cases, treating the underlying cause of hypoxia or long-term oxygen therapy is the preferred treatment for SP. However, phlebotomy can be considered in SP patients, particularly those with symptoms of hyperviscosity. ${ }^{3} \mathrm{We}$ report a patient who presented with erythromelalgia, pruritis, and bleeding but was diagnosed with severe SP. This case illustrates the utility of phlebotomy in SP. We believe that it will help physicians became aware of a rarely used but effective therapeutic modality in SP.

\section{Case Report}

A 60-year-old white male presented to the emergency department for intermittent erythromelalgia that had been occurring for the last year but had increased in frequency. He also had episodes of cyanosis of the lips, facial plethora, conjunctival injection, left upper quadrant pain (LUQ), pruritis unrelated to water, petechia, epistaxis, fatigue, and myalgias. His past medical history was significant for untreated chronic obstructive pulmonary disease (COPD) and recurrent episodes of epistaxis. He was on $81 \mathrm{mg}$ of aspirin (ASA). He had last seen a health care provider 4 years ago.

On examination he was afebrile, heart rate was 64 beats per minute, respiratory rate was 15 respirations per minute, blood pressure was 152/106 $\mathrm{mmHg}$ and oxygen saturation was $83 \%$ on room air. Oxygen saturation improved to $93 \%$ on high flow nasal cannula (HFNC) with FiO2 90\% and 45 LPM. The patient had mild discomfort on LUQ palpation. He was plethoric with conjunctival injection bilaterally, splenomegaly, bilateral pallor of the feet, and petechia to the left lower abdominal region. His physical exam was otherwise unremarkable. His initial hemoglobin was $25.0 \mathrm{~g} / \mathrm{dL}$, hematocrit of $74.6 \%$, white blood cell count of $7,900 / \mu \mathrm{L}$, and platelets of $160,000 / \mu \mathrm{L}$. Peripheral blood smear showed normochromic, normocytic erythrocytosis and polychromasia. Other abnormal lab values included serum bicarbonate of $21 \mathrm{mEq} / \mathrm{L}$ and partial thromboplastin time (aPTT) of 66.2 seconds. His initial venous blood gas (VBG) showed a $\mathrm{pH}$ of 7.36, pCO2 $46 \mathrm{~mm} / \mathrm{Hg}$, and pO2 27 $\mathrm{mm} / \mathrm{Hg}$ while on $38 \% \mathrm{FiO} 2$. He had an otherwise normal basic metabolic panel, hepatic function panel, coagulation panel and urinalysis. Computed topography (CT) of the chest with intravenous contrast (IV) showed no evidence of a pulmonary embolism or aortic dissection but noted underlying bullous emphysema with prominent interstitial markings and ground-glass opacities. The patient was admitted with the diagnoses of hypoxic respiratory failure and polycythemia.

Upon admission, it was unclear if the patient had primary polycythemia or secondary polycythemia in the setting of
Correspondence: Jacob Fuqua, Robert C. Byrd Health Science Center, West Virginia University, PO Box 9160, Morgantown, WV 26506, USA.

E-mail: jacob.fuqua@hsc.wvu.edu

Key words: Polycythemia, Phlebotomy, erythrocytosis.

Contributions: The authors contributed equally.

Conflict of interest: The authors declare no potential conflict of interest.

Funding: None.

Ethics approval: Has been received.

Consent to publish: Has been received.

Availability of data and materials: Available on request.

Received for publication: 24 September 2020. Accepted for publication: 30 April 2021.

This work is licensed under a Creative Commons Attribution-NonCommercial 4.0 International License (CC BY-NC 4.0).

(C) Copyright: the Author(s), 2021

Licensee PAGEPress, Italy

Hematology Reports 2021; 13:8961

doi:10.4081/hr.2021.8961

COPD. The workup for PV revealed an increased EPO level of $32.4 \mathrm{mIU} / \mathrm{mL}$, no mutations to JAK2 V617F, JAK2 Exon 12, or CALR. Serum ferritin was $38 \mathrm{ng} / \mathrm{mL}$, serum iron of $91 \mu \mathrm{g} / \mathrm{dL}$, total iron binding capacity of $335 \mu \mathrm{g} / \mathrm{dL}$, and transferrin of $239 \mu \mathrm{g} / \mathrm{dL}$. He underwent a CT scan of the abdomen and pelvis with IV contrast which was negative for an EPO secreting tumor. The spleen was also found to be of normal size. Transthoracic echocardiography showed a normal left ventricular ejection fraction and no evidence of structural abnormalities or right heart failure. From this initial workup, the patient was diagnosed with polycythemia secondary to chronic hypoxic lung disease. He was continued on HFNC to maintain oxygen saturation above $90 \%$. He was given ASA $81 \mathrm{mg}$ daily, budesonide $80 \mathrm{mcg}$-formoterol 4.5 mcg inhaled twice daily, ipratropium 0.5 mg-albuterol $3 \mathrm{mg}$ by nebulization four times daily as needed for shortness of breath, prednisone $40 \mathrm{mg}$ daily for five days, doxycycline $100 \mathrm{mg}$ daily for 5 days, and enoxaparin $40 \mathrm{mg}$ subcutaneous daily for venous thromboembolism prophylaxis.

Since the patient was symptomatic with a markedly elevated hematocrit and continued need for HFNC due to hypoxia, thera- 
Table 1. Hematocrit level, supplemental oxygen, volume of phlebotomy, and oxygen extraction ratio by hospital day.

\begin{tabular}{|c|c|c|c|c|}
\hline Hospital day & Hematocrit & Supplemental oxygen $\left(\mathrm{FiO}_{2}\right), \%$ & Volume of phlebotomy & Oxygen extraction ratio \\
\hline 1 & 74.6 & 80 & & \\
\hline 2 & 70.1 & 80 & & \\
\hline 3 & 69.7 & 65 & $250 \mathrm{cc}$ & \\
\hline 4 & 68.4 & 80 & $450 \mathrm{cc}$ & \\
\hline 5 & 64.7 & 80 & $450 \mathrm{cc}$ & \\
\hline 6 & 60.2 & 70 & $500 \mathrm{cc}$ & \\
\hline 7 & 57.7 & 60 & & $7 \%$ \\
\hline 8 & 57.8 & 45 & $500 \mathrm{cc}$ & \\
\hline 9 & 54.4 & 36 & & $15 \%$ \\
\hline 10 & 54.8 & 36 & & \\
\hline
\end{tabular}

peutic phlebotomy was initiated on day 3 of admission. Pruritis, erythromelalgia and LUQ pain improved immediately after phlebotomy was initiated but his oxygen requirement did not decline until hospital day 6. Supplemental oxygen requirement, hematocrit, and volume of phlebotomy are displayed in Table 1. Oxygen extraction ratios $\left(\mathrm{O}_{2}\right.$ ER $)$ were used to ensure that phlebotomy was improving oxygen consumption and not resulting in inadequate oxygen delivery to tissue. Arterial oxyhemoglobin level on day 7 was $93.6 \%$, which correlated with the pulse oximeter, and venous oxyhemoglobin level was $86.9 \%$ with an $\mathrm{O}_{2}$ ER of $7 \%$. Due to the low oxygen extraction, phlebotomy was continued. Venous oxyhemoglobin was obtained on hospital day 9 and was found to be $79.2 \%$ with a pulse oximeter reading of $94 \%$ showing an $\mathrm{O}_{2}$ ER $15 \%$. The aPTT also normalized to 29.3 seconds after completion of phlebotomy. The patient was able to be discharged home on $4 \mathrm{~L} / \mathrm{min}$ of supplemental oxygen. ASA $81 \mathrm{mg}$, umeclidinium $62.5 \mathrm{mcg}$ inhaled daily, and budesonide $80 \mathrm{mcg}$-formoterol $4.5 \mathrm{mcg}$ inhaled twice daily were prescribed at discharge. Hematocrit 4 weeks and 8 weeks after discharge was $53.1 \%$ and $49.5 \%$ respectively. No further phlebotomy was performed after discharge.

\section{Discussion and Conclusions}

Polycythemia is defined by a hematocrit elevation greater than $49 \%$ in men and $48 \%$ in women. It can be further categorized as a primary myeloproliferative neoplasm (PV) or secondary to increased EPO levels (SP). $\mathrm{SP}$ is much more common than PV and is most frequently associated with chronic hypoxic states and less commonly EPO producing neoplasms. The symptoms this patient presented with, including erythromelalgia, pruritis, and epistaxis, are more commonly indicative of $\mathrm{PV} .{ }^{4}$
However, elevated serum EPO levels, normal platelet count, and no evidence of genetic mutations along with an obvious history of chronic hypoxic lung disease made SP the most likely diagnosis. This was further supported by continued decrease in hematocrit after prolonged improvement of hypoxia without the need for further phlebotomy. Erythromelalgia is thought to be a microvascular thrombotic process associated with thrombocytosis commonly seen in PV and often responds to ASA and cytoreduction of platelets. ${ }^{5}$ The patient had been taking low dose ASA with no improvement. However, reducing the blood volume resolved the symptoms suggesting that the symptoms were a result of hyperviscosity from a massively increased red cell volume. Bleeding may be seen in patients with PV and is postulated to be a result of acquired von Willebrand Syndrome (avWS). One possibility is that the increased erythrocytosis results in shear stress that destroys the von Willebrand Factor multimer. ${ }^{6}$ Again, with the markedly increased red cell volume, this patient may have had avWS despite having SP and not PV. This is supported by normalization of the aPTT with subsequent phlebotomy.

The mainstay of treatment for SP resulting from chronic hypoxia is to restore adequate oxygenation to tissue. This is done with supplemental oxygen and reversing the primary cause of hypoxia if possible. ${ }^{3}$ Phlebotomy for SP due to hypoxia can be considered in patients with symptomatic hyperviscosity or hematocrit greater than $56 \%$. The British Journal of Haematology endorses a Grade B recommendation to reduce hematocrit to $50-52 \%$ in selected patients with SP due to hypoxic pulmonary disease, but there is concern that reducing the red cell mass may theoretically worsen tissue hypoxia. ${ }^{7}$ However, previous studies have shown that phlebotomy may actually improve oxygen consumption without adversely affecting oxygen delivery in this patient population. ${ }^{8,9}$ This patient continued to have symptoms of hyperviscosity and significant hypoxia requiring high amounts of supplemental oxygen. Once phlebotomy was initiated, his symptoms did improve and supplemental oxygen requirement began to decrease. Using the general rule that a normal $\mathrm{O}_{2} \mathrm{ER}$ is around $25 \%$ at rest, calculation of $\mathrm{O}_{2}$ ER may be beneficial to ensure that phlebotomy is not overly reducing oxygen delivery to tissue which could result in further hypoxic complications. It may also be helpful to reduce the use of excessive supplemental oxygen. ${ }^{9}$

This case of SP in a patient with chronic hypoxic lung disease is unusual because of the markedly elevated hematocrit as well as clinical findings more commonly seen in PV such as erythromelalgia, pruritis, and bleeding. This case also illustrates the utility of phlebotomy in select patients with SP and chronic hypoxic lung disease which many physicians may not be aware of. The use of the oxygen extraction ratio may also be useful for avoiding excessive phlebotomy that may result in tissue hypoxia because of inadequate oxygen delivery and also to limit excessive supplemental oxygen.

\section{References}

1. Parapia LA. History of bloodletting by phlebotomy. Br J Haematol 2008; 143:490-5.

2. Marchioli R, Finazzi G, Specchia G, et al. Cardiovascular events and intensity of treatment in polycythemia vera. New Engl J Med 2020;368 22-33.

3. McMullin MF. Investigation and Management of Erythrocytosis. Curr Hematol Malign Rep 2016;11:342-7.

4. Lee G, Arcasoy MO. The clinical and laboratory evaluation of the patient with erythrocytosis. Eur J Intern Med 2015: 26:297-302. 
5. Michiels JJ. Erythromelalgia and vascular complications in polycythemia vera. Semin Thromb Hemost 1997;23:44154.

6. Mital A, Prejzner W, ŚwiątkowskaStodulska R, Hellmann A. Factors predisposing to acquired von Willebrand syndrome during the course of poly- cythemia vera - retrospective analysis of 142 consecutive cases. Thromb Res 2015;136:754-7.

7. McMullin M, Bareford D, Campbell P, et al. Guidelines for the diagnosis, investigation and management of polycythaemia/erythrocytosis. $\mathrm{Br} \mathrm{J}$ Haematol 2005;130:174-95.
8. Assi TB, Baz E. Current applications of therapeutic phlebotomy. Blood Transf 2014;12:s75-s83.

9. Shah D, Powers S, Bernard H, et al. Increased oxygen uptake following phlebotomy and simultaneous fluid replacement in polycythemic patients. Surgery 1980;88:686-92. 